\section{Visión Electrónica Más que un estado sólido \\ https://doi.org/10.14483/issn.2248-4728}

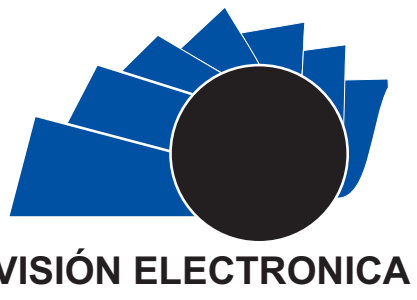

Visión de Caso

\title{
Adquisición de datos Real-Time sobre equipo de protección eléctrica
}

\author{
Real-Time data acquisition on electrical protection equipment \\ Robinson Castillo Méndez', Edgar Mauricio Urquijo Mendoza
}

\section{INFORMACIÓN DEL ARTICULO}

Historia del articulo

Enviado: 19/12/2017

Recibido: 16/03/2018

Aceptado: 26/07/2018

Palabras clave:

Adquisición de datos en tiempo real,

Equipo de protección,

Medición de variables eléctricas,

Redes de distribución de energía.

\section{Keywords:}

Real-Time data acquisition,

Protection equipment,

Electrical variables

measurement,

Electric Power

Distribution Networks.

\section{RESUMEN}

Los sistemas de control empleados en las redes de distribución de energía eléctrica se encuentran en constante evolución; sin embargo, este tipo de sistemas de distribución también presentan constantes desafíos para los operadores, por esto resulta relevante el diseño de controladores de alto desempeño que aumenten las características de robustez, eficiencia y seguridad en las aplicaciones orientadas a tales sistemas. En Colombia, el nivel de generación de nuevo conocimiento en relación al análisis, diagnóstico, diseño y control de estos sistemas actualmente es reducido. Por lo anterior, el grupo de investigación GICS del CEET (Centro de Electricidad, Electrónica y Telecomunicaciones) y la empresa ATA ELECTRIC S.A.S. en el marco de la ejecución de un proyecto conjunto de investigación aplicada, se ha propuesto una metodología para validar la implementación de un sistema que permita mejorar el desempeño en redes de distribución de energía eléctrica, implementando en primera instancia elementos básicos de medición de variables sobre un reconectador eléctrico. Se utiliza una herramienta de adquisición de datos en tiempo real, cuyos resultados son plausibles al realizar variaciones en la carga diseñada enfocadas a la medición de sobre corriente.

\section{ABSTRACT:}

Control Systems used in Electric Power Distribution Networks are constantly evolves, nevertheless, these kind of systems also stand for permanent challenges for the operators of these distribution systems, hence, it is relevant to design high performance controllers that increases robustness, efficiency and security features for applications oriented to these systems. Level of generation of new knowledge related with the analysis, diagnosis, design and control of these systems is currently low in Colombia, hence, The GICS Research Group (as part of CEET - Electricity, Electronics and Telecommunications Center) and ATA ELECTRIC S.A.S. they have proposed to validate a system to improve performance in electricity distribution networks in the future, in a first stage, implementing basic elements of variable measurement in electrical protection equipment. A real-time data acquisition platform is used, the results of which are plausible by making variations in the load focus on the measurement of the current.

\footnotetext{
1 Ingeniero Electrónico, Universidad Distrital Francisco José de Caldas, Colombia. MSc.(c) En Microelectrónica, Universidad de Sevilla, España. Lugar de trabajo: Centro de Electricidad, Electrónica y Telecomunicaciones - CEET, Servicio Nacional de Aprendizaje - SENA, Regional Distrito, Colombia. Correo electrónico rcastillo48@misena.edu.co. ORCID: https://orcid.org/0000-0002-7412-6306.

2 Ingeniero Electrónico, Universidad de los Andes, Colombia. Esp. En Sistemas de Transmisión y Distribución de Energía Eléctrica, Universidad de los Andes, Colombia. Lugar de trabajo: ATA ELECTRIC S.A.S, Colombia. Correo electrónico: gerencia@ataelectric.com. orcID: https://orcid.org/o000-0002-. $\underline{7690-8766 .}$

Citar este artículo como: R. Castillo-Méndez y E. M. Urquijo-Mendoza, "Adquisición de datos Real-Time sobre equipo de protección eléctrica”, Visión electrónica, algo más que un estado sólido, vol. 1, no. 1, Edición especial, enero-junio 2018. DOI revista: https://doi.org/10.14483/issn.2248-4728.
} 


\section{Introducción}

Las técnicas empleadas en las redes de distribución de energía eléctrica se encuentran en constante evolución en lo que se refiere a sus sistemas de control; en este sentido se trata de uno los mayores desafíos para los operadores de sistemas de distribución especialmente al hacer referencia a control centralizado y funcionalidad integrada. La introducción de Tecnologías de la Información y las Comunicaciones (TIC), o de plataformas de Control Industrial en un esfuerzo por "automatizar el sistema de distribución", se constituyen como alternativas para superar esta problemática y potencialmente evolucionar hacia un entorno inteligente a nivel de distribución de energía.

Por otro lado, la automatización es una de las alternativas que la mayor parte de las empresas a nivel mundial han elegido para mantener su competitividad en el mundo globalizado en el que hoy desarrollan sus actividades productivas y de comercialización [1]. Así mismo, en Colombia, es evidente que existen bajos niveles de generación de nuevo conocimiento a nivel local y nacional, relacionados con el análisis, diagnóstico y control de alto desempeño de sistemas eléctricos y mecatrónicos; por lo tanto, resulta relevante el diseño de controladores de alto desempeño que aumenten las características de robustez, eficiencia y seguridad en las aplicaciones [2-3].

Considerando lo anterior, la empresa ATA ELECTRIC S.A.S. ha desarrollado un proyecto conjunto con el grupo de investigación GICS del CEET (Centro de Electricidad, Electrónica y Telecomunicaciones) que aborda esta problemática, por lo que el presente artículo es un producto de la misma y se estructura sobre la medición básica de variables eléctricas para ser aplicadas sobre un reconectador eléctrico, incorporando una herramienta de adquisición de datos en tiempo real.

\section{Materiales y métodos.}

\subsection{Protecciones eléctricas en distribución}

Las protecciones eléctricas son equipos, operaciones o sistemas, que buscan:

- Detectar condiciones anormales o intolerables en el sistema eléctrico y tomar acciones correctivas

- Prevenir daños en equipos, la vida de los seres vivos

- Minimizar la no entrega de energía debida a situaciones de falla
Existen diferentes protecciones para cada elemento del sistema de potencia: generador, líneas, barrajes, transformadores, entre otros. Las principales protecciones en redes de distribución son:

- Relés de sobrecorriente: dispositivos lógicos cuya función es la comparación de una o varias señales de entrada con respecto a una referencia. Si los valores de entrada se desvían de la referencia por encima de un valor de ajuste (Setting) entonces se realiza una acción como disparo (apertura), cierre o alarma.

- Seccionalizadores: elemento que distingue entre faltas permanentes de la red y faltas momentáneas (pasajeras), que desaparecen espontáneamente. El $90 \%$ de las faltas en redes de distribución pertenecen al segundo tipo y, sin embargo, conducen a la actuación de fusibles que abren innecesariamente el circuito, interrumpiendo el servicio. El seccionalizador sólo abre el circuito e interrumpe el servicio en una rama cuando esta acción es absolutamente inevitable.

- Fusibles: dispositivo cuyo principio de interrupción se basa inicialmente en la fusión de un elemento conductor. Una vez iniciado el proceso de fusión, se produce el arco eléctrico dentro del fusible, siendo posteriormente apagado por medio del material de relleno. Todo el fenómeno de interrupción se efectúa sin manifestación externa alguna, por lo que normalmente el usuario no se da cuenta de la energía que ha manejado el fusible. Los principales tipos de fusible de alta capacidad de ruptura son los tipos $\mathrm{NH}, \mathrm{HH}$, cilíndricos, D y DO.

- Reconectadores: dispositivos de protección primaria cuyas características de reconexión mejoran los índices de confiabilidad del sistema debido a la propiedad de discriminar fallas permanentes de temporales, a partir de la duración que estas presentan.

De las anteriores, los Reconectadores Eléctricos son equipos de protección que combinan sensado, procesamiento, interrupción, y recierres en un solo dispositivo [4]. Los reconectadores se usan como dispositivos de protección de líneas de distribución. Además, son equipos que se pueden usar para dar cumplimiento a algunas resoluciones de la CREG (Comisión de Regulación de Energía y Gas).

De otro lado, los Relés son dispositivos que procesan señales que provienen de sensores de corriente y voltaje, con esto opera contactos físicos de acuerdo a cierta 
lógica programada. Los principales tipos de relé para protección en distribución son:

- Relés de protección: detecta defectos y condiciones anormales, generalmente dispara interruptores además de dar alarma.

- Relés de monitoreo: verifica condiciones en el sistema de potencia o en el sistema de protección; no ordena abrir interruptores.

- Relés de recierre: establece una secuencia de cierre del interruptor luego de su disparo por protección; al recierre automático se lo denomina reconexión automática.

- Relés de regulación: se activa cuando parámetros operativos se desvían de los límites predeterminados; usualmente trabaja vinculado al control de equipos.

- Relés auxiliares: opera como consecuencia de las señales de apertura o cierre de un circuito para complementar la acción de otro relé o dispositivo.

- Relés de sincronización: asegura las condiciones apropiadas para la interconexión de dos secciones de un sistema eléctrico.

Vale la pena indicar que los relés no realizan directamente las maniobras de conexión y desconexión del sistema eléctrico en caso de falla o sobrecorriente, esta labor la realiza un equipo de corte y maniobra.

\subsection{Plataforma de adquisición de datos}

Los controladores embebidos en tiempo real, en general, ofrecen una potente ejecución embebida autónoma para aplicaciones Real-Time. Muchos de estos controladores están diseñados para extrema robustez, confiabilidad y bajo consumo de energía, con entradas de alimentación de 9 a 35 VDC que suministran energía aislada a los módulos que se implementen y un rango de temperatura de operación promedio de -40 a $70^{\circ} \mathrm{C}$.

Por ejemplo, el NI cRIO-9022 de National Instruments, es uno de los controladores más potentes, cuenta con un procesador en tiempo real de $533 \mathrm{MHz}$ y proporciona un muy alto nivel de rendimiento en esa línea de productos de National Instruments. "Los controladores NI cRIO$901 x$ cuentan con un procesador industrial de $400 \mathrm{MHz}$ que equilibra el bajo consumo de energía y el poderoso análisis, control y registro en tiempo real de las señales de punto flotante" [5].
Herramientas como LabVIEW ${ }^{\circledR}$, que es un potente y versátil entorno de programación gráfica, fue desarrollado principalmente para facilitar el control de instrumentación y adquisición y análisis de datos. Por lo tanto, tiene varias características clave que lo convierten en una buena opción en un entorno de control y automatización [6], aclarando que no es la única plataforma o herramienta que puede emplearse para aplicaciones Real-Time.

\subsection{Sistemas de control}

La tecnología empleada en los sistemas de automatización han experimentado una evolución generacional, donde en una primera generación se emplearon elementos mecánicos para reemplazar la fuerza humana por máquinas, posteriormente, los sistemas neumáticos aparecieron dada la naturaleza de las señales de control y los mecanismos principalmente mecánicos existentes en ese contexto. Una siguiente generación se basa en las señales eléctricas, de voltaje, como tecnología para los sistemas de control. Sistemas de cuarta generación utilizan tecnología electrónica digital para sus sistemas de control, la Figura 1 muestra esta evolución, [3]

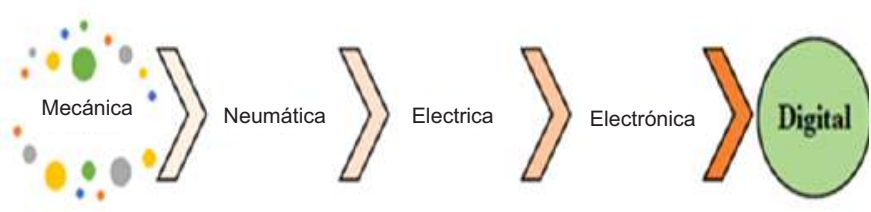

Figura 1. Evolución tecnología en sistemas de automatización [3].

Los sistemas de control han experimentado grandes cambios, los avances tecnológicos han permitido una gran capacidad de almacenamiento de datos, mejores sistemas de comunicación, conectividad, mayores velocidades y el desarrollo de sistema de control multidisciplinario [3].

\subsection{Medición de variables}

Los equipos de protección eléctrica presentan diferentes parámetros de entrada dependiendo de su robustez y aplicación específica, en el caso de los Relés de monitoreo variables de entrada como voltaje, corriente, frecuencia, potencia, presión o temperatura son fundamentales para establecer los esquemas de control necesarios en el caso de sobrecorrientes de tiempo e instantáneos, sobrevoltajes o bajos voltajes en el sistema. 


\section{Alternativa propuesta.}

El sistema propuesto para la realización de mediciones sobre un equipo de protección eléctrica y posterior verificación del desempeño en redes de distribución de energía eléctrica se ilustra en la figura 2. El sistema fundamenta su funcionamiento en plataformas como las de National Instruments ${ }^{\circledR}$ y su capacidad para implementar elementos de medición y control embebidos para aplicaciones en tiempo real.

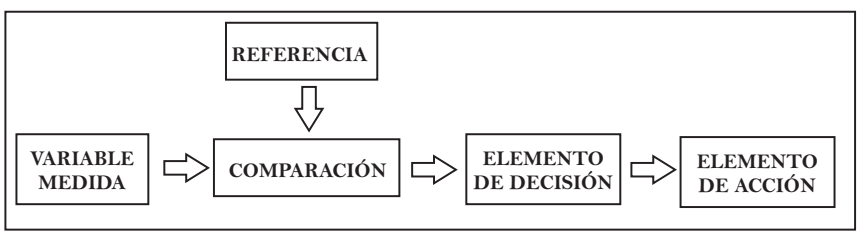

Figura 2. Diagrama sistema propuesto.

Fuente: elaboración propia.

Las principales variables consideradas para el sistema han sido el voltaje y la corriente, las mediciones se realizaron empleando un sistema de adquisición en tiempo real, sin embargo, en esta primera fase el trabajo realizado se efectuó en ambiente de laboratorio para lo cual se acondicionó una carga variable trifásica a 120 voltios $\mathrm{AC}$ y corriente máxima de 37 amperios $\mathrm{AC}$ por fase.

Una vez acondicionada esta carga variable, con el software de programación asociado a la plataforma de adquisición de datos en tiempo real, se han desarrollado los cuatro primeros bloques mostrados en la figura 2.

En un primer bloque del sistema, la señal adquirida es mostrada en tiempo real un una gráfica tiempo-voltaje y tiempo-corriente, así mismo un indicador muestra la magnitud de la señal adquirida, su frecuencia y su fase.

Basándose en la Normativa de Protecciones para sobre corriente y las curvas de tiempo inverso - TCC (Característica Tiempo - Corriente), las cuales relacionan la corriente y el tiempo en una curva de protección, se han establecido los niveles en el sistema para las funciones 50 y 51 (IEEE) que sirven de referencia para la posterior comparación de estos niveles.

Sin embargo, para el desarrollo del sistema a nivel de laboratorio, estos niveles de corriente se fijaron en un máximo de 30 Amperios y a partir de ese punto se ha simulado sobre corriente y observado y ajustado los tiempos de reacción para posterior protección del sistema, así quedó establecida para esta primera etapa la referencia de corriente.
La figura 3 ilustra el principio del elemento de comparación a un nivel básico para el tercer bloque, allí se realiza una comparación de niveles de tensión de acuerdo a los parámetros establecidos, los cuales en el posterior sistema real aplicará para las funciones $50 \mathrm{y}$ 51 , y en esta etapa experimental inicial para los establecidos como referencia de acuerdo a las entrada simuladas de voltaje y corriente presentes en las entradas analógicas del sistema.

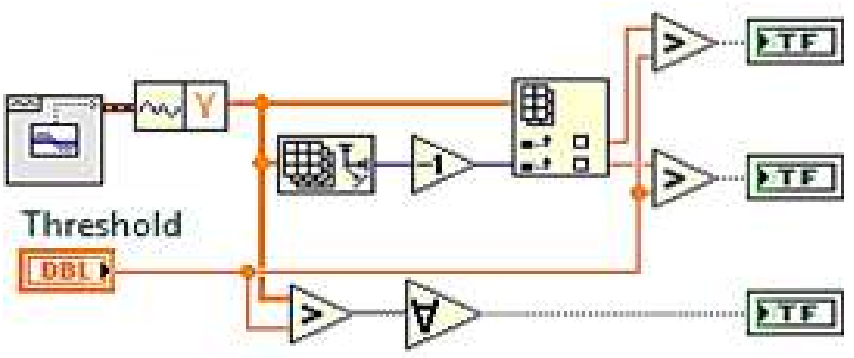

Figura 3. Principio básico del elemento comparador de voltaje. Fuente: elaboración propia

De acuerdo a los valores obtenidos en la etapa de comparación, una señal de acción es enviada al respectivo actuador para la ejecución de la acción de control, que en este caso, tratándose de un relé, se basa en abrir o cerrar parte del circuito de la red.

El elemento de acción, correspondiente al cuarto bloque descrito en la figura 2, se encuentra fuera del ambiente de laboratorio empleado, pues esta función es asumida por un equipo de corte y maniobra. El relé en este caso como elemento de decisión realiza una apertura o cierre que activa una alarma visual que indica ocurrencia de falla por inconsistencia respecto a la referencia ajustable establecida.

\section{Resultados}

Algunos resultados obtenidos al realizar variaciones en la carga diseñada para estas pruebas enfocadas a la medición de sobre corriente se ilustran en la tabla 1:

\begin{tabular}{|c|c|c|}
\hline REFERENCIA & $\begin{array}{c}\text { CORRIENTE } \\
\text { PRUEBA }\end{array}$ & $\begin{array}{c}\text { TIEMPO } \\
\text { DISPARO (S) }\end{array}$ \\
\hline $5 \mathrm{~A}$ & $5,5 \mathrm{~A}$ & 0,15 \\
\hline $10 \mathrm{~A}$ & $12 \mathrm{~A}$ & 0.15 \\
\hline $15 \mathrm{~A}$ & $15,2 \mathrm{~A}$ & 0.13 \\
\hline $20 \mathrm{~A}$ & $20,1 \mathrm{~A}$ & $0,1_{6}$ \\
\hline $25 \mathrm{~A}$ & $26 \mathrm{~A}$ & 0,14 \\
\hline $30 \mathrm{~A}$ & $37 \mathrm{~A}$ & $0,1^{2}$ \\
\hline
\end{tabular}

Tabla 1. Tiempo de disparo de acuerdo a nivel de comparación sobre corriente Fuente: elaboración propia 
Como se indicó anteriormente, debido a que un relé de protección es un dispositivo lógico cuya función es la comparación de una o varias señales de entrada con respecto a una referencia, la corriente es la variable más utilizada para la detección de anomalías en los elementos del sistema eléctrico -dado el elevado incremento que se registra en su valor cuando se presentan fallas-

Como se observa en la tabla 1, todo valor que excede la corriente normal de operación de un dispositivo se considera sobre corriente, de acuerdo a la función que se quiera realizar, el tiempo de respuesta puede ser ajustable, en este caso se consideró el sobrepaso del nivel de referencia para un tiempo estándar en estos sistemas por debajo de 0,2 segundos para generar algún tipo de acción.

\section{Conclusiones}

El trabajo presentado constituye la primera fase de la validación de un sistema orientado a mejorar el desempeño de las redes de distribución de energía eléctrica, dicho sistema está soportado por una plataforma robusta de desarrollo de sistemas embebidos, sistemas de control y automatización.

De otro lado, esta fase de la investigación representa la base de un trabajo orientado al desarrollo de tecnologías que faciliten la localización de fallas, aislamiento y restauración del servicio de las redes de distribución de energía eléctrica en Colombia; este tipo de sistemas, además de sus respectivo sistema de control, involucran sistemas automatización, switches, redes de comunicación, reconectadores, relés y sistemas de gestión para la distribución, en este caso se ha propuesto incorporar tecnología de adquisición de datos en tiempo real para observar si hay lugar a mejora en la respuesta del sistema y robustez del mismo.

Así mismo, mediante este primer producto se busca contribuir a la solución de la problemática identificada en Colombia relacionada con el bajo nivel de generación de nuevo conocimiento en relación al análisis, diagnóstico, diseño y control de estos sistemas. respuesta del sistema y robustez del mismo.

\section{Referencias}

[1] CIMAV, "Diagnóstico y Prospectiva de la Mecatrónica en México”, Chihuahua, México,
2008.

[2] A. Zidan, M. Khairalla, A. M. Abdrabou, T. Khalifa, K. Shaban, A. Abdrabou, R. El Shatshat y A. M. Gaouda, "Fault Detection, Isolation, and Service Restoration in Distribution Systems: State-of-the-Art and Future Trends", IEEE Trans. Smart Grid, vol. 8, n o. 5, 2016, p p. $1-16$. DOI.

https://doi.org/10.1109/TSG.2016.2517620.

[3] Y. V. P. Kumar y R. Bhimasingu, "Key Aspects of Smart Grid Design for Distribution System Automation: Architecture and Responsibilities", Procedia Technol., vol. 21, 2015 , p p. $352-359$. D O I : https://doi.org/10.1016/j.protcy.2015.10.047.

[4] IEEE, "IEEE Guide for Automatic Reclosing of Circuit Breakers for AC Distribution and Transmission Lines", IEEE Std C37.1042012, pp. 1-72. DOI.

https://doi.org/10.1109/IEEESTD.2012.6232 $\underline{415}$.

[5] I. Vesely, M. Sir y D. Zmecnik, "Problems of SITL: Testing the simulink model by Compact RIO", IFAC Proceedings Volumes, vol. 45, no. 7, 2012, pp. 388-392. DOI: https://doi.org/10.3182/20120523-3-CZ3015.00074 .

[6] L. Wang, Y. Tan, X. Cui y H. Cui, "The application of LabVIEW in data acquisition system of solar absorption refrigerator," Energy Procedia, vol. 16, 2011, pp. 1496-1502. D O I .

https://doi.org/10.1016/j.egypro.2012.01.235. 